PROCEEDINGS OF THE

AMERICAN MATHEMATICAL SOCIETY

Volume 49, Number 2, June 1975

\title{
PRACTICALLY PERFECT THREE-MANIFOLD GROUPS ${ }^{1}$
}

\author{
D. R. McMILLAN, JR.
}

ABSTRACT. Let $M^{3}$ be a 3-manifold containing no 2-sided projective plane. Let $G \neq\{1\}$ be a finitely-generated subgroup of $\pi_{1}\left(M^{3}\right)$ such that $G$ is indecomposable relative to free product, and such that $G$ abelianized is finite. ( $G$ is "practically perfect".) Then, it is shown that there is a compact 3-submanifold $Z^{3} \subset M^{3}$ such that $\pi_{1}\left(Z^{3}\right)$ contains a subgroup of finite index conjugate to $G$, and $Z^{3}$ is bounded by a 2 -sphere. Some related extensions of this result are given, plus an application to compact absolute neighborhood retracts in 3-manifolds.

1. Introduction. Several authors [5], [8], [1] have obtained results concerning conditions under which the fundamental group of a 3-manifold $M^{3}$ can have elements of finite order. The best result in this direction is D. B. A. Epstein's Theorem 8.2 of [1], which says that a finite subgroup $G$ of $\pi_{1}\left(M^{3}\right)$ can arise only in one of two ways: either $G \cong Z_{2}$ and $G$ "conjugates" into a 2sided projective plane in $M^{3}$, or $M^{3}$ has a connected sum factor with finite fundamental group and $G$ conjugates into this factor. Our aim here is to obtain some analogous results in the case that $G / G^{\prime}$ is finite. ( $G^{\prime}$ denotes the commutator subgroup of $G$.)

There are some new things to note in our context, however (as contrasted to [1]). First, finiteness of $G / G^{\prime}$ does not prevent $G$ from being a nontrivial free product. (A finite group is indecomposable relative to free product.) Secondly, the nonorientable case is trickier since, even if $G / G^{\prime}$ is finite and $G$ is indecomposable relative to free product, a subgroup of index two in $G$ may not inherit these properties. The second difficulty seems more serious, and has led us routinely to assume that $M^{3}$ contains no 2-sided projective planes.

The basic tools (Theorems 1 and 1') lean heavily on Epstein's results.

Presented to the Society, January 26, 1973 under the title Uncovering some properties of the fundamental group; received by the editors June 28, 1973 and, in revised form, January 9, 1974 and March 19, 1974.

AMS (MOS) subject classifications (1970). Primary 57A65, 55A05, 55C15; Second ary $55 \mathrm{~A} 35$.

Key words and phrases. Fundamental group, 3-manifold, connected sum, free product, absolute neighborhood retract, aspherical, perfect group.

1This research was supported in part by NSF grant GP-29537X1. 
Theorem 2 is our " $G / G$ ' finite" analog of his theorem about finite subgroups, and we assume in it that the group $G$ in question is *-indecomposable (i.e., indecomposable relative to free product, which is denoted by $*$ ). Theorem 3 is the best we have been able to do when $G$ is not assumed $*$-indecomposable. Finally, Theorem 4 is a geometrically-oriented application of these results to certain compact absolute neighborhood retracts in 3-manifolds. The following result may be easily deduced from either Theorem 2 or Theorem 4, and the reader may wish-to take it as an exercise: If $X$ is a compact, connected absolute neighborhood retract in $E^{3}$ ( $E^{n}$ denotes Euclidean $n$-space) and the homology group $H_{1}(X ; Z)$ is finite, then $X$ is simply connected.

All mappings, manifolds, submanifolds, etc. are to be taken in the piecewise-linear sense. A "manifold" is connected. If $M^{3}$ is a 3-manifold, then $\hat{M}^{3}$ is the 3-manifold obtained by attaching a 3-cell to each 2-sphere in its boundary, $\partial M^{3}$. A space $X$ is aspherical if $\pi_{n}(X)=0$ for $n \geq 2$. A 3-manifold $M^{3}$ is irreducible if each 2-sphere in $M^{3}$ bounds a 3-cell in $M^{3}$. A good reference for 3-manifold terminology is [7]. We use $\Delta^{n}$ to denote an $n$-simplex, $I=\Delta^{1}$, and $S^{n}$ is $\partial \Delta^{n+1}$. Real projective $n$-space is $P^{n}$. Homeomorphism of spaces $A, B$ is symbolized $A \approx B$; and the fact that groups $G, H$ are isomorphic is symbolized $G \cong H$. The infinite cyclic group is $Z$; the cyclic group of finite order $n$ is $Z_{n}$. Homology groups are singular and have $Z$ coefficients unless otherwise indicated.

2. Surrounding a subgroup. We collect in one theorem some results of Epstein [1] for later reference.

Theorem 1. Let $M^{3}$ be a 3-manifold that possibly has boundary, but that contains no 2-sided projective plane. Let $G$ be a subgroup of $\pi_{1}\left(M^{3}\right)$ such that $G$ is *-indecomposable and not infinite cyclic. Suppose that either

(a) $G$ has torsion, or

(b) $G$ is isomorphic to the fundamental group of a closed 3-manifold.

Then there is a compact 3-submanifold $Z^{3} \subset$ Int $M^{3}$ such that $\partial Z^{3}$ is a 2-sphere and $G$ is conjugate in $\pi_{1}\left(M^{3}\right)$ to a subgroup of finite index in $\pi_{1}\left(Z^{3}\right)$. In case $(\mathrm{a}), \pi_{1}\left(Z^{3}\right)$ is finite and $Z^{3}$ is orientable.

Proof. If $G$ has torsion, then it contains a finite subgroup $H$ with more than one element. By [1, Theorem 8.2] there is a compact 3-submanifold $Z^{3}$ $C$ Int $M^{3}$ such that $\partial Z^{3} \approx S^{2}, \pi_{1}\left(Z^{3}\right)$ is finite, and $H$ is conjugate to a subgroup of $\pi_{1}\left(Z^{3}\right)$. ( $Z^{3}$ is necessarily orientable.) By the Kurosh subgroup theorem [4, p. 243], $G$ is conjugate to a subgroup of $\pi_{1}\left(Z^{3}\right)$ or to a subgroup 
of $\pi_{1}\left(M^{3}-\right.$ Int $\left.Z^{3}\right)$. Since $H \neq\{1\}$, the former must hold.

Suppose now that $G \neq\{1\}$ is torsion-free but that $G \cong \pi_{1}\left(N^{3}\right)$, where $N^{3}$ is closed. Then $N^{3}$ contains no projective plane. Thus, since $\pi_{1}\left(N^{3}\right)$ is not $\cong Z$ and is $*$-indecomposable, it follows from Epstein's version [1, Theorem 1.1] of the sphere theorem that $\pi_{2}\left(N^{3}\right)=0$. Further, $\pi_{1}\left(N^{3}\right)$ is infinite, so that $N^{3}$ is aspherical by [1, Lemma 8.1]. The existence of the required 3-submanifold $Z^{3}$ is then a consequence of [1, Theorem 8.8].

There is a variation on Theorem 1 which some may prefer. (Cf. also the definition of "splittable group" given before Theorem 3 and used in Theorems 3 and 4.)

Theorem 1'. Let $M^{3}$ be a 3-manifold that possibly has boundary, but that contains no 2-sided projective plane. Let $G$ be a subgroup of $\pi_{1}\left(M^{3}\right)$ such that $G$ is *-indecomposable, and not infinite cyclic. Suppose that either

(a) $G$ has torsion, or

(b) $H_{3}\left(G ; Z_{2}\right) \neq 0$ and $G$ embeds in $\pi_{1}\left(N^{3}\right)$ for some compact 3-manifold $N^{3}$.

Then there is a compact 3-submanifold $Z^{3} \subset$ Int $M^{3}$ such that $\partial Z^{3}$ is a 2-sphere and $G$ is conjugate in $\pi_{1}\left(M^{3}\right)$ to a subgroup of finite index in $\pi_{1}\left(Z^{3}\right)$. In case $(a), \pi_{1}\left(Z^{3}\right)$ is finite and $Z^{3}$ is orientable.

The proof of Theorem $1^{\prime}$ is similar to that of Theorem 1, and exploits the ideas of $\$ 7$ of [1]. The proof is left to the reader.

Theorem 2. Let $M^{3}$ be a 3-manifold that possibly has boundary, but that contains no 2-sided projective plane. Let $G \neq\{1\}$ be a finitely-generated subgroup of $\pi_{1}\left(M^{3}\right)$ such that $G$ is *-indecomposable, and $G / G^{\prime}$ is finite. Then there is a compact 3-submanifold $Z^{3} \subset$ Int $M^{3}$ such that $\partial Z^{3}$ is a 2 sphere and $G$ is conjugate in $\pi_{1}\left(M^{3}\right)$ to a subgroup of finite index in $\pi_{1}\left(Z^{3}\right)$. (It follows that $H_{1}\left(Z^{3}\right)$ is finite, and that $Z^{3}$ is orientable.)

Proof. By Scott [6], $G$ is finitely-presented. We may thus assume without loss of generality that $M^{3}$ is compact. It suffices (by Theorem 1) to show that if $G$ is torsion-free, then $G$ is isomorphic to the fundamental group of a closed 3-manifold. By Theorem 4 of [3] (cf. also [2, Theorem 4.4], which contains an alternate proof), $G \cong \pi_{1}\left(N^{3}\right)$ for some compact 3-manifold $N^{3}$. If $G$ is torsion-free, then $\partial N^{3}$ contains no projective plane and hence by Lemma 2.4 of [2], $\partial N^{3}$ consists entirely of 2-spheres. Then $\hat{N}^{3}$ is the required closed 3-manifold. Verifying the parenthetical remark is left as an exercise for the reader. 
Let us say that a finitely-generated group $G$ is splittable if $G$ has no infinite cyclic free factor, and if each free factor of $G$ that is $*$-indecomposable either has torsion, or is isomorphic to the fundamental group of a closed 3-manifold. For example, it suffices to know that $G$ is the fundamental group of a 3-manifold and has finite abelianization. (Cf. also Theorem 1'.)

Theorem 3. Suppose that $S$ is an arcwise-connected Hausdorff space and $U$ is an open, connected set in $S$ such that $U$ is also an open 3-manifold containing no 2-sided projective plane. Let $G$ be a finitely-generated group that is splittable, $G \subset \pi_{1}(U)$. Suppose that there does not exist a compact 3-submanifold $Z^{3} \subset U$ with $\partial Z^{3}$ a 2-sphere, and two distinct free factors of $G$ such that each factor is conjugate in $\pi_{1}(U)$ to a subgroup of finite index in $\pi_{1}\left(Z^{3}\right)$. Then the inclusion-induced homomorphism $\pi_{1}(U) \rightarrow \pi_{1}(S)$ maps $G$ monomorphically.

Proof. Write $G=G_{1} * \cdots * G_{n}$, where each $G_{i} \neq\{1\}$ is $*$-indecomposable. Then each $G_{i}$ is finitely-generated, none is isomorphic to $Z$, and each $G_{i}$ either has torsion or is isomorphic to the fundamental group of a closed 3-manifold. Then repeated application of Theorem 1 (keeping in mind our special "conjugacy" hypothesis about the $G_{i}$ 's) yields disjoint compact 3-submanifolds $Z_{1}, \cdots, Z_{n}$ such that each $\partial Z_{i} \approx S^{2}$ and for each $i, G_{i}$ is conjugate in $\pi_{1}(U)$ to a subgroup of finite index in $\pi_{1}\left(Z_{i}\right)$.

Now fix a basepoint $p \in U-\cup Z_{i}$ for $\pi_{1}(U)$. Then by van Kampen's theorem and the fact that $S$ is Hausdorff (so that $S-\bigcup Z_{i}$ is open in $S$ ),

$$
\pi_{1}(S, p)=\pi_{1}\left(Z_{1}\right) * \cdots * \pi_{1}\left(Z_{n}\right) * \pi_{1}\left(S-\bigcup Z_{i}, p\right)
$$

where the embedding of $\pi_{1}\left(Z_{i}\right)$ in $\pi_{1}(S, p)$ is determined by translating $\pi_{1}\left(Z_{i}\right)$ to the basepoint along the $i$ th arc in a disjoint collection of arcs in $U-U$ Int $Z_{i}$. Let $k$ be the inclusion $U \rightarrow S$. Then since $G_{i}$ is conjugate in $\pi_{1}(U, p)$ to a subgroup of finite index in $\pi_{1}\left(Z_{i}\right), k_{*}$ maps each $G_{i}$ monomorphically into $\pi_{1}(S, p)$.

By Scott's Theorem 1.7 of [6], the fact that $k_{*}$ maps $G$ monomorphically will follow once it is shown that the subgroup

$$
J=\operatorname{Image}\left[k_{*}: G \rightarrow \pi_{1}(S, p)\right]
$$

has at least $n$ free factors. If $J$ had fewer than $n$ free factors, some ( $*$-indecomposable, $¥ Z$ ) free factor $J_{1}$ of $J$ would contain conjugates (in $J$ ) of two distinct groups $k_{*}\left(G_{i}\right)$, say $k_{*}\left(G_{1}\right)$ and $k_{*}\left(G_{2}\right)$. But then, recalling the displayed expression for $\pi_{1}(S, p)$ above, we see that $J_{1}$ would be conjugate in 
$\pi_{1}(S, p)$ to a subgroup of exactly one of the free factors displayed there. This is impossible, since a subgroup $\neq\{1\}$ of one free factor cannot also be conjugate in $\pi_{1}(S, p)$ to a subgroup of another free factor. The result follows.

Example. Let $M^{3}$ be the compact, orientable 3-manifold-with-boundary obtained from real projective 3-space $P^{3}$ by removing the interior of a 3-cell, then adding an orientable 1 -handle to the resulting 2 -sphere boundary. Thus,

$$
\pi_{1}\left(M^{3}\right) \cong Z_{2} * Z \cong \pi_{1}\left(\operatorname{Int} M^{3}\right) .
$$

Let $J \subset \partial M^{3}$ be a simple closed curve representing (up to conjugacy) the generator $t$ of the $Z$ factor of $\pi_{1}\left(M^{3}\right)$. Let $S$ be obtained by adding a 2handle to $M^{3}$ along $J$, so that $\pi_{1}(S) \cong Z_{2}$. If $U=$ Int $M^{3}$ and $G$ is the subgroup of $\pi_{1}(U)$ generated by $Z_{2}$ and $t^{-1} Z_{2} t$, then $G \cong Z_{2} * Z_{2}$ (see Problem 10 , p. 194 of [4]), yet the inclusion-induced homomorphism $\pi_{1}(U) \rightarrow \pi_{1}(S)$ does not map $G$ monomorphically. Of cour'se, the "conjugacy" hypothesis of Theorem 3 is violated.

Theorem 4. Let $M^{3}$ be a 3-manifold that possibly has boundary, but that contains no 2-sided projective plane. Suppose that $X \subset M^{3}$ is a connected compact absolute neighborhood retract whose fundamental group $\pi_{1}(X)$ is splittable. (For example, suppose that $H_{1}(X)$ is finite.) Then each sufficiently tight connected neighborhood $U$ of $X$ in $M^{3}$ contains a connected neighborhood $V$ of $X$ in $M^{3}$ such that each loop in $V-X$ is contractible in $U$.

Proof. We may assume that $M^{3}$ is an open 3-manifold. Let $U$ be any connected neighborhood of $X$ in $M^{3}$ that retracts onto $X$. Let $V$ be any connected neighborhood of $X$ in $U$ that admits a strong deformation retraction in $U$ onto $X$. (That is, there is a map $h: V \times I \rightarrow U$ such that, letting $h_{t}(x)=h(x, t)$ and identifying $V \times\{0\}$ with $V$, we have: $h_{0}=$ inclusion $V$ $\rightarrow U$, each $h_{t} \mid X=$ inclusion $X \rightarrow U$, and $h_{1}$ retracts $V$ onto $X$. ) Write $\pi_{1}(X)=G_{1} * \cdots * G_{n}$, where each $G_{i} \neq\{1\}$ is $*$-indecomposable. Then each $G_{i}$ is finitely-generated, no $G_{i}$ is isomorphic to $Z$, and each $G_{i}$ either has torsion or is isomorphic to the fundamental group of a closed 3-manifold.

We claim that each loop in $V-X$ is contractible in $U$. Let $L: \partial \Delta^{2} \rightarrow$ $V-X$ be given. (We put $|L|=L\left(\partial \Delta^{2}\right)$.) Let $V_{1}$ be a connected neighborhood of $X$ in $V-|L|$ that admits a strong deformation retraction in $V-|L|$ onto $X$. Let $i, j$ denote the inclusions $X \rightarrow V_{1}, V_{1} \rightarrow V-|L|$, respectively.

We note first that there is no compact 3-submanifold $Z^{3} \subset V_{1}$ such that $\partial Z^{3} \approx S^{2}$ and such that (say) each of $i_{*}\left(G_{1}\right)$ and $i_{*}\left(G_{2}\right)$ is conjugate in 
$\pi_{1}\left(V_{1}\right)$ to a subgroup of finite index in $\pi_{1}\left(Z^{3}\right)$. (Observe that $\pi_{1}\left(Z^{3}\right) \nsubseteq Z$, and that $\pi_{1}\left(Z^{3}\right)$ is $*$-indecomposable.) For

$$
j_{*} \pi_{1}\left(Z^{3}\right) \subset j_{*} \pi_{1}\left(V_{1}\right)=j_{*} i_{*} \pi_{1}(X) \cong G_{1} * \cdots * G_{n} .
$$

Thus, the denial of our assertion would imply that each of $j_{*} i_{*}\left(G_{1}\right)$ and $j_{*} i_{*}\left(G_{2}\right)$ is conjugate in $\pi_{1}(V-|L|)$ to a subgroup of the same one of $j_{*} i_{*}\left(G_{1}\right), \cdots, j_{*} i_{*}\left(G_{n}\right)$ into which $j_{*} \pi_{1}\left(Z^{3}\right)$ conjugates. Since $j_{*} i_{*} \pi_{1}(X)$ is a retract of $\pi_{1}(V-|L|)$, this is impossible.

We can now verify our earlier claim by an application of Theorem 3 . Specifically, let $S$ be the space obtained by attaching a 2-cell to $U$ along $L$, and let the present $V_{1}$ be the distinguished open set considered in the statement of Theorem 3. Then $L$ is freely homotopic in $U$ to a loop in $X$, and hence to a loop $\bar{L}$ in $V_{1}$ representing an element of $i_{*} \pi_{1}(X)$. By Theorem 3 , the inclusion-induced homomorphism $\pi_{1}\left(V_{1}\right) \rightarrow \pi_{1}(S)$ maps $i_{*} \pi_{1}(X)$ monomorphically. Since $\bar{L}$ is contractible in $S$, it follows that $\bar{L}$ is contractible in $V_{1}$. Hence $L$ is contractible in $U$, as desired.

\section{REFERENCES}

1. D.B.A. Epstein, Projective planes in 3-manifolds, Proc. London Math. Soc. (3) 11 (1961), 469-484. MR 27 \#2968.

2. D. E. Galewski, J. G. Hollingsworth and D. R. McMillan, Jr., On the fundamental group and homotopy type of open three-manifolds, General Topology and Its Appl. 2 (1972), 299-313. MR 47 \#5880.

3. William Jaco, Fintely-presented subgroups of three-manifold groups, Invent. Math. 13 (1971), 335-346. MR 45 \#9325.

4. W. Magnus, A. Karrass and D. Solitar, Combinatorial group theory: Presentations of groups in terms of generators and relations, Pure and Appl. Math., vol. 13, Interscience, New York, 1966. MR 34 \#7617.

5. C. D. Papakyriakopoulos, On Dehn's lemma and the asphericity of knots, Ann. of Math. (2) 66 (1957), 1-26. MR 19, 761.

6. G. P. Scott, Finitely-generated 3-manifold groups are finitely-presented, J. London Math. Soc. (2) 6 (1973), 437-440.

7. F. Waldhausen, On irreducible 3-manifolds which are sufficiently large, Ann. of Math. (2) 87 (1968), 56-88. MR 36 \#7146.

8. J. H. C. Whitehead, On 2-spheres in 3-manifolds, Bull. Amer. Math. Soc. 64 (1958), 161-166.

DEPARTMENT OF MATHEMATICS, UNIVERSITY OF WISCONSIN, MADISON, WISCON- 